\title{
Supplemente für Kognitionserhalt ungeeignet
}

\author{
Nahrungsergänzungsmittel sind bei vielen Menschen beliebt und werden in der Laienpresse \\ für ihre präventiven Effekte gefeiert. Die wissenschaftlichen Daten sind hingegen oft ernüch- \\ ternd. Ein aktuelles Beispiel liefert eine größere US-amerikanische Studie.
}

_ Aus Beobachtungsstudien gibt es viele Hinweise, dass eine ungünstige Ernährungsweise das Risiko für die Entwicklung einer Alzheimer-Demenz erhöht. Im Fokus stehen dabei eine hohe Aufnahme von gesättigten Fettsäuren und ein niedriger Gemüsekonsum. In der groß angelegten prospektiven AgeRelated Eye Disease Study 2 (AREDS2) wurde neben Fragen zur Makuladegeneration auch der Effekt von Nahrungsergänzungsmitteln auf kognitive Funktionen untersucht. 3.741 Probanden (89\% der Gesamtpopulation) beteiligten sich an dieser Nebenstudie.

Über die Studiendauer von fünf Jahren erhielten alle Teilnehmer eine tägliche Basissupplementation mit Vitamin C, Vitamin E, Beta-Carotin und Zink. Die Intervention bestand aus der Zufuhr von $1 \mathrm{~g}$ langkettigen, mehrfach ungesät- tigten Fettsäuren und/oder einer Kombination aus $10 \mathrm{mg}$ Lutein und $2 \mathrm{mg}$ Zeaxanthin. Diese beiden Carotinoide kommen in der Natur stets gemeinsam vor und werden als Nahrungsergänzungsmittel verkauft. Die Kontrollgruppe erhielt Placebo.

Die Teilnehmer waren zu Beginn der fünfährigen Studie durchschnittlich 72,7 Jahre alt und litten an einer altersbedingten Makuladegeneration. Zu Studienbeginn und danach alle zwei Jahre wurden sie telefonisch mehreren kognitiven Tests unterzogen.

Die Ergebnisse zeigten, dass keine der durchgeführten Interventionen die kognitiven Fähigkeiten signifikant positiv beeinflusste.

- Chew EY, Clemons TE, Agrón E et al. Effect of Omega-3 Fatty Acids, Lutein/Zeaxanthin, or Other Nutrient Supplementation on Cognitive Function: The AREDS2 Randomized Clinical Trial. JAMA. 2015;314:791-801

\section{KOMMENTAR}

Die Autoren betonen, dass die Aussagekraft der Studie begrenzt ist und lediglich bedingt Rückschlüsse auf die eingesetzten Supplemente möglich sind. Die Aussage, dass ein gesunder Lebensstil einer Alzheimer-Erkrankung vorbeugen kann, ist damit nicht widerlegt. Vielmehr scheint es stärker auf eine lebenslange gesundheitsförderliche Lebensweise anzukommen als auf die Zufuhr von Nahrungsergänzungsmitteln im Alter. Künftige Studien sollten daher Ernährungsmuster untersuchen. AREDS2 hat zwar in den Medien für Aufmerksamkeit gesorgt, sollte aber nicht überbewertet werden. Die Ergebnisse sind wertvoll, im Hinblick auf das untersuchte Kollektiv mit relativ hohem Altersdurchschnitt und mindestens einer chronischen Erkrankung allerdings auch nicht unbedingt überraschend.

Dr. rer. nat. C. Holzapfel

\section{Röntgenbild zeigt einen kolonartigen Megaösophagus}
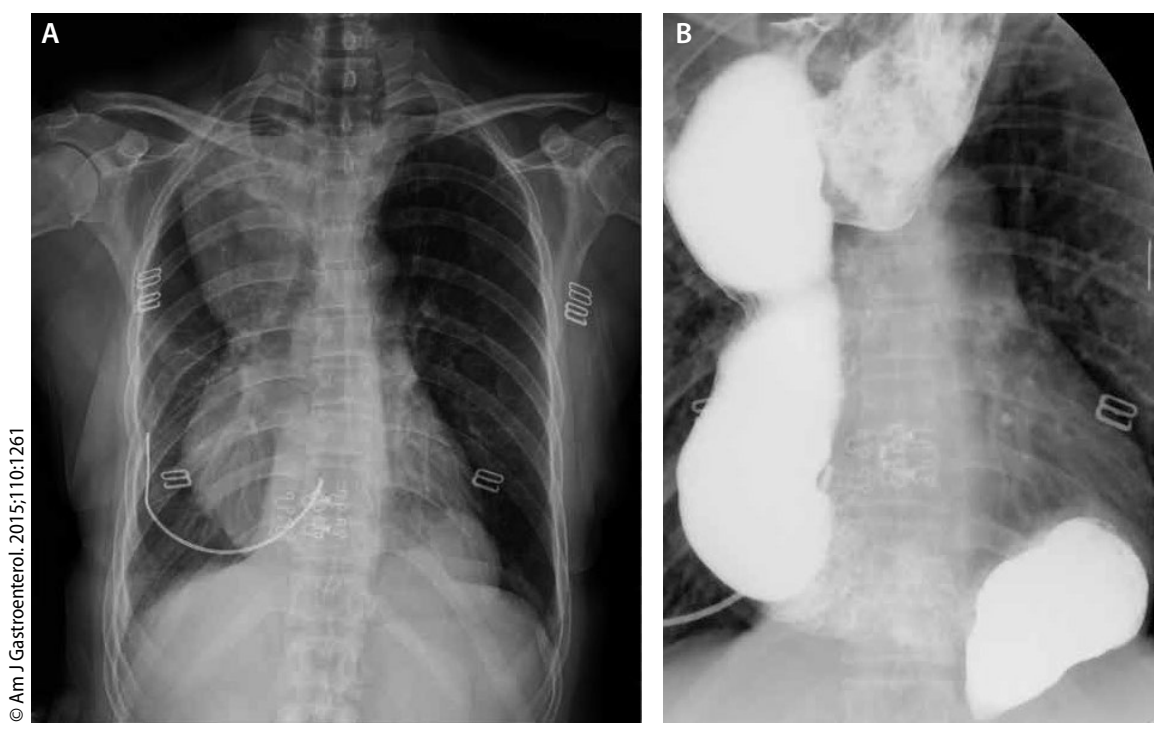

A: Deutliche Verbreiterung des Mediastinums im Röntgenthorax; B: Extrem dilatierter, geschlängelter Ösophagus, sichtbar gemacht per Bariumbreischluck.
Eine 48-jährige Frau aus China litt seit 20 Jahren unter zunehmenden Schluckbeschwerden bei festen und flüssigen Speisen. Sie musste nach dem Essen häufig erbrechen. Im Röntgenthorax stellte sich eine ausgeprägte Verbreiterung des Mediastinums dar (Abb. A). Ein Bariumbreischluck (Abb. B) brachte die Klärung: Die Frau hatte einen extrem dilatierten und geschlängelten Ösophagus, der sich mindestens im Durchmesser des Kolons darstellte. An der Kardia lief das Organ spitz zu. Die Entleerung des Bariumbreis war massiv verzögert.

Wir erfahren nicht, ob der Fall eher die extreme Leidensfähigkeit der chinesischen Landbevölkerung oder die schlechte medizinische Versorgung illustriert. Es wird auch nicht mitgeteilt, ob sich die Frau operieren ließ.

Prof. Dr. med. H. S. FüeßI

- Lin F et al. Colon-like Megaesophagus. Am J Gastroenterol. 2015;110:1261 